\title{
Oral Administration of Oleuropein and Its Semisynthetic Peracetylated Derivative Prevents Hepatic Steatosis, Hyperinsulinemia, and Weight Gain in Mice Fed with High Fat Cafeteria Diet
}

\author{
Saverio Massimo Lepore, ${ }^{1}$ Valeria Maria Morittu, ${ }^{1}$ Marilena Celano, ${ }^{1}$ \\ Francesca Trimboli, ${ }^{1}$ Manuela Oliverio, ${ }^{1}$ Antonio Procopio, ${ }^{1}$ Carla Di Loreto, ${ }^{2}$ \\ Giuseppe Damante, ${ }^{2}$ Domenico Britti, ${ }^{1}$ Stefania Bulotta, ${ }^{1}$ and Diego Russo ${ }^{1}$ \\ ${ }^{1}$ Department of Health Sciences, University "Magna Graecia" of Catanzaro, Campus "S. Venuta", Viale Europa, \\ Germaneto, 88100 Catanzaro, Italy \\ ${ }^{2}$ Department of Medical and Biological Sciences, University of Udine, Piazzale Kolbe 4, 33100 Udine, Italy
}

Correspondence should be addressed to Stefania Bulotta; bulotta@unicz.it and Diego Russo; d.russo@unicz.it

Received 10 September 2015; Revised 26 November 2015; Accepted 30 November 2015

Academic Editor: Małgorzata Kotula-Balak

Copyright (C) 2015 Saverio Massimo Lepore et al. This is an open access article distributed under the Creative Commons Attribution License, which permits unrestricted use, distribution, and reproduction in any medium, provided the original work is properly cited.

\begin{abstract}
The high consumption of olive tree products in the Mediterranean diet has been associated with a lower incidence of metabolic disorders and cardiovascular diseases. In particular, the protective effects of olive oil have been attributed to the presence of polyphenols such as oleuropein (Ole) and its derivatives. We have synthesized a peracetylated derivative of Ole (Ac-Ole) which has shown in vitro antioxidant and growth-inhibitory activity higher than the natural molecule. In this study, male C57BL/6JOlaHsd mice were fed with a standard (std), cafeteria (caf) diet, and caf diet supplemented with Ole $(0.037 \mathrm{mmol} / \mathrm{kg} / \mathrm{day})$ and Ac-Ole $(0.025 \mathrm{mmol} / \mathrm{kg} /$ day $)$ for 15 weeks. We observed a significant reduction in the caf diet-induced body weight gain and increase of abdominal adipose tissue. Also, Ole and Ac-Ole prevented the development of hepatic steatosis. Finally, Ole and Ac-Ole determined a lower increase of HDL and LDL-cholesterol levels and corrected caf diet-induced elevation of plasma glucose concentrations by improving insulin sensitivity. The observed beneficial properties of Ole and Ac-Ole make these compounds and in particular Ac-Ole promising candidates for a potential pharmaceutic use in metabolic disorders.
\end{abstract}

\section{Introduction}

Dietary consumption of olive oil is considered a key component to explain the association of Mediterranean diet with a lowered incidence of metabolic disorders and cardiovascular diseases [1-3]. The beneficial action of extra virgin olive oil is mainly attributed to its seco-phenolic compounds and their secondary metabolites, which have highly been investigated as both whole extracts and individual components $[4,5]$. In particular, the proactive ingredient oleuropein (Ole) and its derivative hydroxytyrosol (HT) have demonstrated several beneficial effects in in vitro and in vivo experimental models [6-9]. Ole is a natural polyphenolic compound belonging to the secoiridoids and is present in high amount in the leaves and unprocessed olive drupes. After crushing, Ole is hydrolyzed by endogenous esterase giving rise to its aglycone derivate that is the active secoiridoid present in virgin olive oil [8]. After oral administration, it is rapidly absorbed with maximum plasma concentration occurring $2 \mathrm{~h}$ after administration and excreted in urine mainly as glucuronides or as free form in very low concentrations $[10$, 11].

The ability of Ole and Ole derivatives to scavenge reactive oxygen species and their antioxidant activity $[6,8,12,13]$ have been associated with the protection against cardiovascular diseases and metabolic disorders [14-17]. Two other mechanisms have been proposed to explain the hypoglycemic effect of Ole and HT: the improvement of glucose-induced insulin 
TABLE 1: Composition of the standard and cafeteria diets.

\begin{tabular}{lcc}
\hline & STD & CAF \\
\hline Energy density (kcal/g) & 3.1 & 4.9 \\
\hline Protein (\%) & 18.6 & 8.4 \\
\hline Fat (\%) & 6.2 & 26.0 \\
of which saturated (\%) & 0.9 & 14.8 \\
\hline Carbohydrate (\%) & 44.2 & 52.7 \\
of which sugar (\%) & - & 21.5 \\
\hline Fiber (\%) & 3.5 & 1.8 \\
\hline Salt (\%) & 0.2 & 0.3 \\
\hline Iron (\%) & 0.02 & 0.004 \\
\hline Calcium (\%) & 1.0 & 0.2 \\
\hline Potassium (\%) & 0.6 & 0.2 \\
\hline Phosphorus (\%) & 0.7 & 0.2 \\
\hline Zinc (\%) & 0.007 & 0.001 \\
\hline B1 (\%) & 0.0017 & 0.0003 \\
\hline B2 (\%) & 0.0015 & 0.0003 \\
\hline
\end{tabular}

release and the increased peripheral uptake of glucose [14, 18]. Moreover, an effect of Ole on insulin action and secretion has been described in overweight middle-aged men, who received a diet supplemented with olive leaf polyphenols. Such an effect was independent by fat distribution, dietary intakes, and physical activity and was similar to that obtained with antidiabetic drug treatment [19]. In addition, Ole has been successfully used to prevent hepatic steatosis in mice fed with high fat diet [9, 20-22].

In our laboratory, we have synthesized a peracetylated derivative of Ole (Ac-Ole) in good yields and very mild conditions by applying nonconventional synthetic methodologies on the natural substrate Ole extracted from renewable raw material $[23,24]$. In previous works, Ac-Ole has shown in vitro higher antioxidant and antiproliferative effects on thyroid and breast cancer cells than the natural molecule $[25,26]$.

A relevant model to study metabolic disorders in mice is the highly palatable cafeteria (caf) diet: this dietary intervention is closer to Western food habits than the traditional high fat diet, creating therefore a phenotype of exaggerated obesity with glucose intolerance and inflammation [27].

In the present study, we analyzed the effects of oral subchronic administration of Ole and Ac-Ole in C57BL/ $6 \mathrm{JOlaHsd}$ mice fed with a caf diet.

\section{Methods}

2.1. Diet Composition and Compounds. The nutritional compositions of the standard (std) diet (Teklad Global 18\% Protein Rodent Diet, Harlan Laboratories s.r.l., Udine, Italy) and the caf diet are reported in Table 1. Caf diet is a mixture of std diet and commercial food as snack, chocolate, biscuits, and wafers. Ole (MW 540, dissolved in water at the dose of $0.037 \mathrm{mmol} / \mathrm{kg}$, equivalent to $20 \mathrm{mg} / \mathrm{kg}$ ) and its semisynthetic derivative Ac-Ole (MW 792, dissolved in corn oil at the dose of $0.025 \mathrm{mmol} / \mathrm{kg}$, equivalent to $20 \mathrm{mg} / \mathrm{kg}$ ) were obtained as described [23].

2.2. Animals and Treatment. Male C57BL/6JOlaHsd mice ( $n=20$, aged 4 weeks) were purchased from Harlan Laboratories s.r.l., housed in a temperature $\left(20-22^{\circ} \mathrm{C}\right)$ and humidity (64\%) controlled animal room and maintained on a $12: 12 \mathrm{~h}$ light/dark cycle. After 2 weeks of quarantine the mice were divided into four separate groups $(n=5$ in each group) of equal average body weight $(19.4 \pm 1.2 \mathrm{~g})$ : mice fed with a std diet (named STD), mice fed with a caf diet (named CAF), mice fed with a caf diet supplemented with daily oral administration of Ole $(0.037 \mathrm{mmol} / \mathrm{Kg}$; named OLE), and mice fed with a caf diet supplemented with daily oral administration of Ac-Ole $(0.025 \mathrm{mmol} / \mathrm{Kg}$; named Ac-OLE). A std diet (Harlan Laboratories s.r.l.), suggested for this strain of mice, was used as control to (a) analyze the effects of caf diet and (b) investigate the effects of treatment with Ole and Ac-Ole. Animals of all groups received the same vehicle used for the peracetylated compound and had free access to food and water. Every day, Ole and Ac-Ole were prepared freshly before administration and added to caf diet pellet: it was checked that each mouse had ingested the food containing the daily dose of Ole and Ac-Ole. Food intake was calculated subtracting every week the aliquot of food not ingested by the total food added to each cage. Body weight, nasoanal (N-A) length, and girth waist of all animals were recorded at weekly interval. At the end of treatment, after overnight fasting, mice were deeply anesthetized by intraperitoneal injection of tiletamine hydrochloride and zolazepam hydrochloride (Zoletil, Virbac, France) at dosage of $80 \mathrm{mg} / \mathrm{kg}$, and medetomidine hydrochloride (Domitor, Orion Corporation, Finland) at dosage of $6.6 \mathrm{mg} / \mathrm{kg}$. Blood samples were obtained by cardiac venipuncture and collected into blood collection tubes (Vacuette, Greiner Bio-One GmbH Bad Haller Str. 32, 4550 Kremsmunster, Austria) containing $\mathrm{K}_{3}$-EDTA. The whole liver, abdominal fat, thoracic aorta, kidney, and lung were removed and weighed and then were washed in normal saline solution and immediately fixed in 10\% neutral buffered formalin (Sigma-Aldrich s.r.l., Milan, Italy). All efforts were made to minimize the number of animals used in this study and their suffering.

2.3. Biochemical Analysis. Blood samples, collected at the end of the treatments, were centrifuged at $1700 \mathrm{~g}$ for $10 \mathrm{~min}$ at room temperature and serum was stored at $-20^{\circ} \mathrm{C}$ until use. By using commercial reagents (Siemens Healthcare Diagnostics s.r.l., Milan, Italy) and an automated biochemistry analyzer (Dimension EXL, Siemens Healthcare Diagnostics s.r.l.) the levels of basal glucose, triglycerides, total cholesterol, low density lipoproteins cholesterol (LDL), high density lipoproteins cholesterol (HDL), alanine aminotransferase (ALT), and aspartate aminotransferase (AST) were determined. The concentration of insulin and leptin was measured using ELISA kits (Rat/Mouse Insulin ELISA Kit; Mouse Leptin ELISA Kit, EMD Millipore Corporation, Darmstadt, Germany) according to the manufacturers' instructions. Approximate insulin resistance (IR) was calculated using the homeostasis 


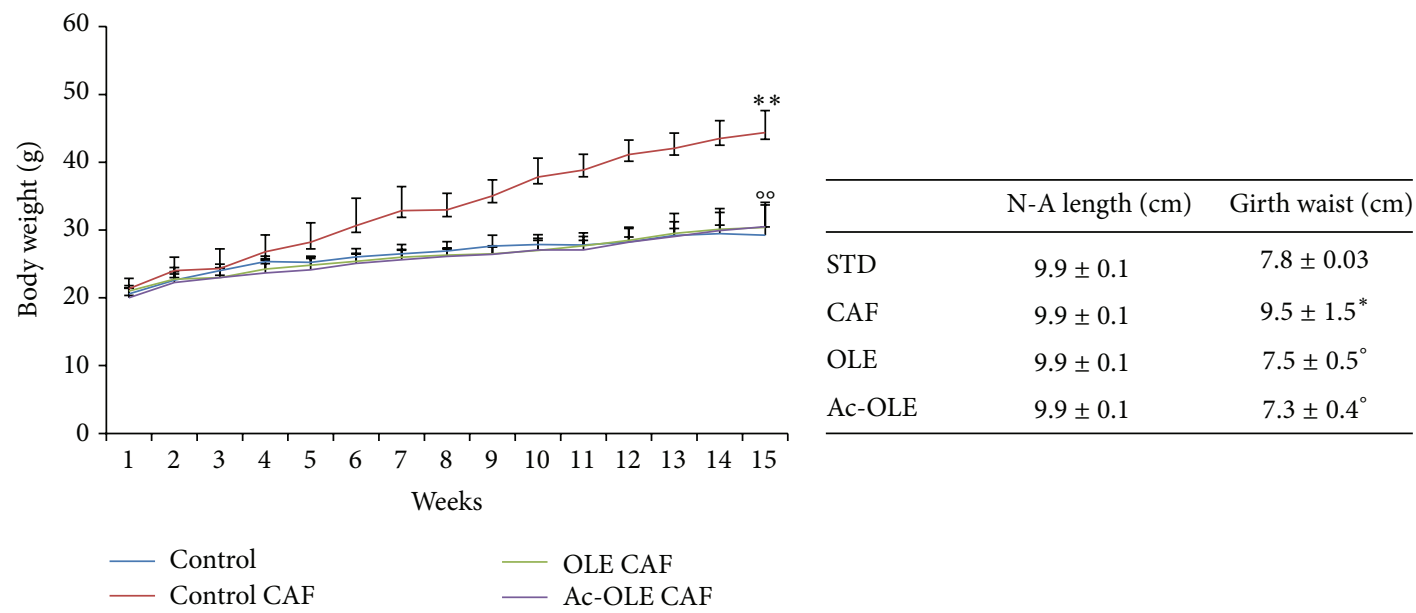

(a)

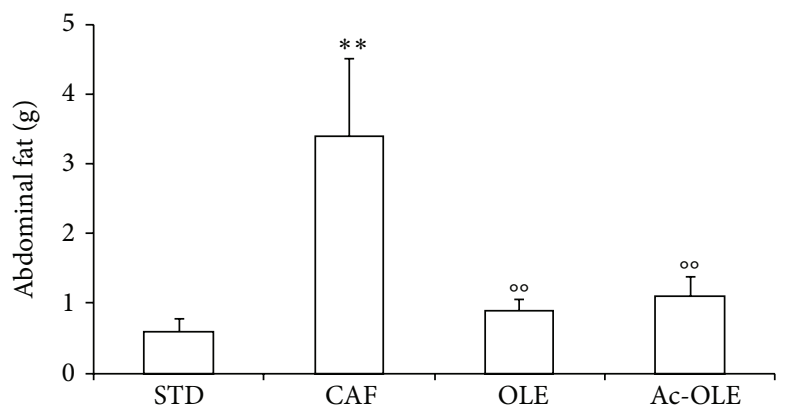

(c)

FIGURE 1: Effects of Ole and Ac-Ole on body weight and girth waist. (a) Body weight and (b) N-A length and girth waist are relative to the end of treatment (15 weeks). (c) Abdominal fat tissue weight. Values are mean \pm SD. ${ }^{*} P<0.05$ and ${ }^{* *} P<0.01$ compared to mice fed with std diet; ${ }^{\circ} \mathrm{P}<0.05$ and ${ }^{\circ} \mathrm{P}<0.01$ compared to mice fed with caf diet.

model assessment (HOMA)-IR using the following formula: [glucose $(\mathrm{mmol} / \mathrm{L}) \times$ insulin $(\mu \mathrm{U} / \mathrm{mL})] / 22.5[28]$.

\subsection{Histopathological Study. Tissue samples were embedded} in paraffin after dehydration through a graded ethanol series followed by xylene. Several sections (thickness 4$5 \mu \mathrm{m}$ ) from each paraffin block were cut, mounted on slides and, after deparaffinization and rehydration, were stained with haematoxylin and eosin according to standard methods for histological assessment under the light microscopy. In liver of each mouse, steatosis was evaluated by counting the percentage of vacuolated cells. For each liver two different sections were evaluated, each of them cut along the major axis of the gland. All cells of each section were evaluated. The Gomori trichrome stain was used to detect fibrosis [29].

2.5. Statistical Analysis. Results are expressed as mean \pm standard deviation (SD). The data relative to body weight, naso-anal length, girth waist, food consumption, energy intake, liver weight, and hematological and clinical chemistry parameters were analyzed using Sigma Plot version 12 (Systat Software, Inc.). One-way ANOVA followed by Tukey multiple comparison test was performed for different treatment groups. Differences between groups were considered significant at $P<0.05$.

\section{Results}

3.1. Ole and Ac-Ole Prevented Increase in Body and Abdominal Fat Weight. Mice were fed for 15 weeks with std, caf, or caf diet supplemented with Ole or Ac-Ole. During the experimental period, the mice showed no signs of suffering and no significant differences appeared in hematological parameters at the end of the treatments (Table 2). The body weight increased in all groups, in accordance with the physiological growth of the mice (Figure 1(a)). In CAF mice, this increase was significantly higher than STD (Figure $1(\mathrm{a}), P<0.01$ ), in parallel with an enhanced abdominal girth (Figure 1(b), $P<0.05)$ and an increase in abdominal fat (Figure 1(c), $P<0.01)$. Supplementation of caf diet with Ole or Ac-Ole reduced the body weight increase (Figure 1(a), $P<0.01$ ), as well as both abdominal girth and final abdominal fat (Figures 1(b) and 1(c), $P<0.05$ and $P<0.01$, resp.). The food intake did not differ among the four groups during the experimental period, whereas a significant increase of energy intake was produced by caf diet (Table $3, P<0.001$ ), and the addition of the two compounds did not cause significant changes in OLE 
TABLE 2: Effects of Ole and Ac-Ole on hematological parameters.

\begin{tabular}{lcccccc}
\hline Parameter & Units & STD & CAF & OLE & Ac-OLE & Range \\
\hline WBC & $\left(10^{3} / \mathrm{mm}^{3}\right)$ & $2.4 \pm 0.5$ & $2.8 \pm 0.8$ & $3.8 \pm 1.2$ & $4.0 \pm 1.0$ & $2.3-7.7$ \\
RBC & $\left(10^{6} / \mathrm{mm}^{3}\right)$ & $8.6 \pm 1.1$ & $9.6 \pm 0.2$ & $9.0 \pm 0.3$ & $9.3 \pm 0.4$ & $12.4-10.2$ \\
HGB & $(\mathrm{g} / \mathrm{dL})$ & $13.4 \pm 0.2$ & $14.1 \pm 0.5$ & $12.4 \pm 0.4$ & $43 \pm 0.4$ & $44.3 \pm 2.1$ \\
HCT & $(\%)$ & $41.9 \pm 6.3$ & $46.5 \pm 2.1$ & $43.7 \pm 1.5$ & $47.4 \pm 0.6$ \\
MCV & $(\mathrm{fL})$ & $48.3 \pm 1.2$ & $48.3 \pm 1.5$ & 14.6 \\
MCH & $(\mathrm{pg})$ & $14.6 \pm 0.1$ & $14.7 \pm 0.4$ & $13.8 \pm 0.2$ & $13.7 \pm 0.1$ & 14.9 \\
MCHC & $(\%)$ & $30.4 \pm 0.7$ & $30.4 \pm 0.5$ & $28.4 \pm 0.4$ & $29.0 \pm 0.4$ & $28.6-30.8$ \\
Platelets & $\left(10^{3} / \mu \mathrm{L}\right)$ & $1183.2 \pm 71.4$ & $1097.0 \pm 316.8$ & $1170.2 \pm 160.0$ & $1041.2 \pm 317.7$ & $980-1390$ \\
Neutrophils & $(\%)$ & $7.4 \pm 1.8$ & $13.1 \pm 6.4$ & $9.8 \pm 1.8$ & $11.4 \pm 2.1$ & $2-34$ \\
Lymphocytes & $(\%)$ & $87.6 \pm 1.1$ & $81.4 \pm 7.3$ & $86.2 \pm 2.3$ & $80.9 \pm 5.6$ & $1.3 \pm 1.7$ \\
Monocytes & $(\%)$ & $0.8 \pm 0.2$ & $1.4 \pm 0.9$ & $1.0 \pm 0.2$ & $2.4-97$ \\
Eosinophils & $(\%)$ & $1.6 \pm 0.5$ & $2.1 \pm 0.8$ & $1.4 \pm 0.8$ & $2.3 \pm 1.4$ \\
\hline
\end{tabular}

Blood samples were collected at the end of treatments and analysed using Hematology Analyzer ADVIA 2120 (Siemens Healthcare Diagnostics s.r.l., Milan, Italy) to obtain hematology parameters. STD: mice fed with STD diet; CAF: mice fed with CAF diet; OLE = mice fed with CAF diet plus OLE at $0.037 \mathrm{mmol} / \mathrm{kg} / \mathrm{day}$; Ac-OLE: mice fed with CAF diet plus Ac-OLE at $0.025 \mathrm{mmol} / \mathrm{kg} /$ day. WBC: white blood cells; RBC: red blood cells; HGB: hemoglobin; HCT: hematocrit; MCV: mean corpuscular volume; MCH: mean corpuscular hemoglobin; MCHC: mean corpuscular hemoglobin concentration.

TABLE 3: Effects of Ole and Ac-Ole on food and energy intake.

\begin{tabular}{lcccc}
\hline & STD & CAF & OLE & Ac-OLE \\
\hline Food intake (g/week) & $20.8 \pm 0.8$ & $22.8 \pm 2.8$ & $22.1 \pm 2.4$ & $21.6 \pm 2.1$ \\
Energy intake (kcal/week) & $64.5 \pm 2.6$ & $103.0 \pm 9.2^{* * *}$ & $101.2 \pm 10.8^{* * *}$ & $99.9 \pm 6.2^{* * *}$ \\
\hline
\end{tabular}

STD: mice fed with STD diet; CAF: mice fed with CAF diet; OLE = mice fed with CAF diet plus Ole at $0.037 \mathrm{mmol} / \mathrm{kg} / \mathrm{day} ;$ Ac-OLE $=$ mice fed with CAF diet plus Ac-Ole at $0.025 \mathrm{mmol} / \mathrm{kg} /$ day. Values are mean $\pm \mathrm{SD} .{ }^{* * *} P<0.001$ versus STD.

and Ac-OLE-treated groups when compared to CAF group (Table 3).

\subsection{Ole and Ac-Ole Treatment Prevented Lipid Accumulation} in Liver and Other Tissues. Liver weight was significantly increased in CAF $(1.6 \pm 0.4 \mathrm{~g})$ versus STD $(1.1 \pm 0.1 \mathrm{~g})$. Treatment with Ole and Ac-Ole prevented hepatic enlargement, as evident by the weight values, both absolute and normalized for the body weight, similar to those of the STD mice (Figures 2(a) and 2(b)). Histologic analysis of liver tissue sections representative of the four groups of animals is shown in Figure 2(c) and the quantitation of steatosis is reported in Figure 2(d): caf diet induced a significant steatosis, which was greatly reduced in OLE and Ac-OLE groups. In terms of inflammatory cell infiltration, no significant difference was detectable among the four groups of animals; indeed, in all mice only a mild and very focal lymphocytic infiltration in the portal triad was detectable. Gomori staining indicates the absence of fibrosis in all four groups of animals (data not shown). Caf diet induced no detectable differences in the other examined tissues (Figure 3).

3.3. Effects of Ole and Ac-Ole on Serum Lipid Levels. Caf fed mice showed significantly higher levels of plasma total, HDL, and LDL cholesterol than the std fed mice (Table 4). Ole and Ac-Ole supplemented caf diet determined a lower increase of such levels and, interestingly, LDL-cholesterol levels were maintained in the normal range described for these animals. No significant differences were observed in the levels of plasmatic triglycerides (Table 4). In addition, caf diet induced an elevation in ALT and AST levels when compared with STD group. Following treatment with Ole, only the ALT levels returned to the values of the STD, while Ac-Ole determined a reduction of both enzymes (Table 4). However, transaminase serum levels remained in the normal range in all four groups of mice.

3.4. Ole and Ac-Ole Reduced Glucose, Insulin, and Leptin Levels as well as Insulin Resistance Induced by Caf Diet. Fasting glucose levels were slightly but significantly higher in CAF animals compared to mice fed with std diet, although still in the normal range (Figure 4). Instead, OLE and Ac-OLE groups showed values similar to STD (Figure 4). Interestingly, insulin levels were significantly higher in CAF mice as compared to STD group (Figure $4, P<0.01$ ), while, in both OLE and Ac-OLE animals, they were significantly lower compared to the CAF group (Figure $4, P<0.01$ ). HOMAIR, used as measure of insulin sensitivity, indicated that CAF group showed statistically significant increase in insulin resistance compared to STD group (Figure 4, $P<0.001$ ) while the supplementation of Ole and Ac-Ole on caf diet reversed this effect (Figure 4, $P<0.001$ versus CAF). Also leptin content was significantly changed in CAF as compared to STD mice (Figure 4, $P<0.01$ ); again, lower levels were observed in the groups treated with Ole or its peracetylated derivative (Figure 4, $P<0.01$ versus $\mathrm{CAF}$ ). 
TABLE 4: Effects of Ole and Ac-Ole on serum parameters.

\begin{tabular}{|c|c|c|c|c|c|c|}
\hline Parameter & Units & STD & CAF & OLE & Ac-OLE & Range \\
\hline Cholesterol & $\mathrm{mg} / \mathrm{dL}$ & $108.7 \pm 6.2$ & $183.0 \pm 13.4^{* * *}$ & $158.2 \pm 23.4^{* *}$ & $158.5 \pm 18.5^{* *}$ & $43.7-126.2$ \\
\hline $\mathrm{HDL}$ & $\mathrm{mg} / \mathrm{dL}$ & $94.7 \pm 3.8$ & $136.0 \pm 7.5^{* *}$ & $121.0 \pm 12.3$ & $123.2 \pm 19.5^{*}$ & $51.1-102.5$ \\
\hline LDL & $\mathrm{mg} / \mathrm{dL}$ & $14.0 \pm 2.7$ & $47.0 \pm 7^{* * *}$ & $29.3 \pm 4.7^{* * \circ}$ & $30.7 \pm 3.4^{* * \circ \circ}$ & $13.8-31.0$ \\
\hline Triglycerides & $\mathrm{mg} / \mathrm{dL}$ & $47.0 \pm 8.9$ & $52.6 \pm 3.5$ & $39.0 \pm 6.5$ & $57.5 \pm 6.5$ & $47.1-254.9$ \\
\hline ALT & $\mathrm{U} / \mathrm{L}$ & $36.5 \pm 1.9$ & $41.7 \pm 2.2^{*}$ & $28.7 \pm 0.9^{\circ 00}$ & $31.0 \pm 7.8^{000}$ & $13.3-80.2$ \\
\hline AST & $\mathrm{U} / \mathrm{L}$ & $97.7 \pm 11.1$ & $169.3 \pm 10.7^{* * *}$ & $128.8 \pm 7.4^{\circ}$ & $90.0 \pm 8.5^{000}$ & $40.8-510.6$ \\
\hline
\end{tabular}

STD: mice fed with STD diet; CAF: mice fed with CAF diet; OLE: mice fed with CAF diet plus Ole at $0.037 \mathrm{mmol} / \mathrm{kg} /$ day; Ac-OLE: mice fed with CAF diet plus Ac-Ole at $0.025 \mathrm{mmol} / \mathrm{kg} / \mathrm{day}$; ALT: alanine aminotransferase; AST: aspartate aminotransferase; HDL: high density lipoproteins; LDL: low density lipoproteins. Reference range calculated on the data published in Mouse Database Phenomena (Jackson Laboratory) (http://phenome.jax.org). Values are mean \pm SD. ${ }^{*} P<0.05,{ }^{* *} P<0.01$, and ${ }^{* * *} P<0.001$ compared to STD $;{ }^{\circ} P<0.05,{ }^{\circ 0} P<0.01$, and ${ }^{\circ 00} P<0.001$ compared to CAF.

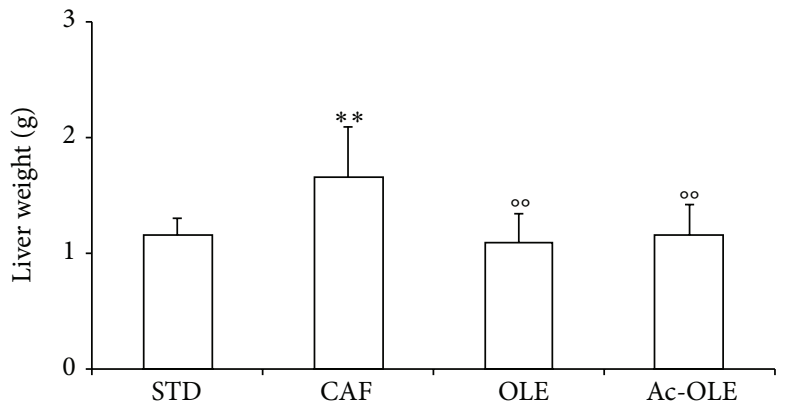

(a)
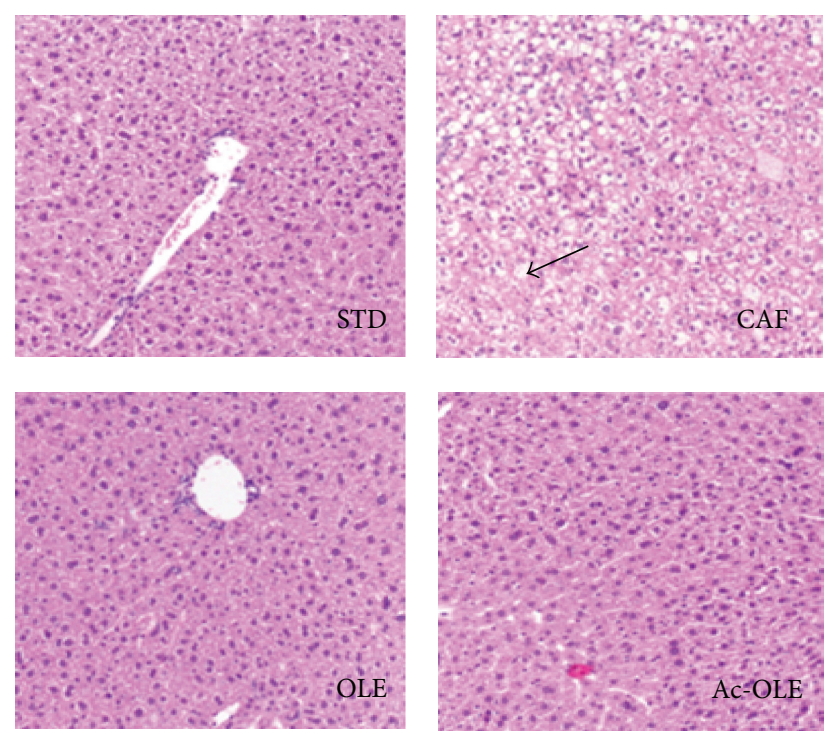

(c)

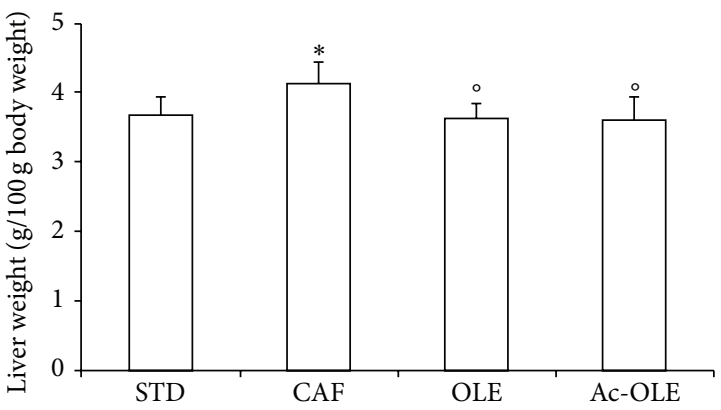

(b)

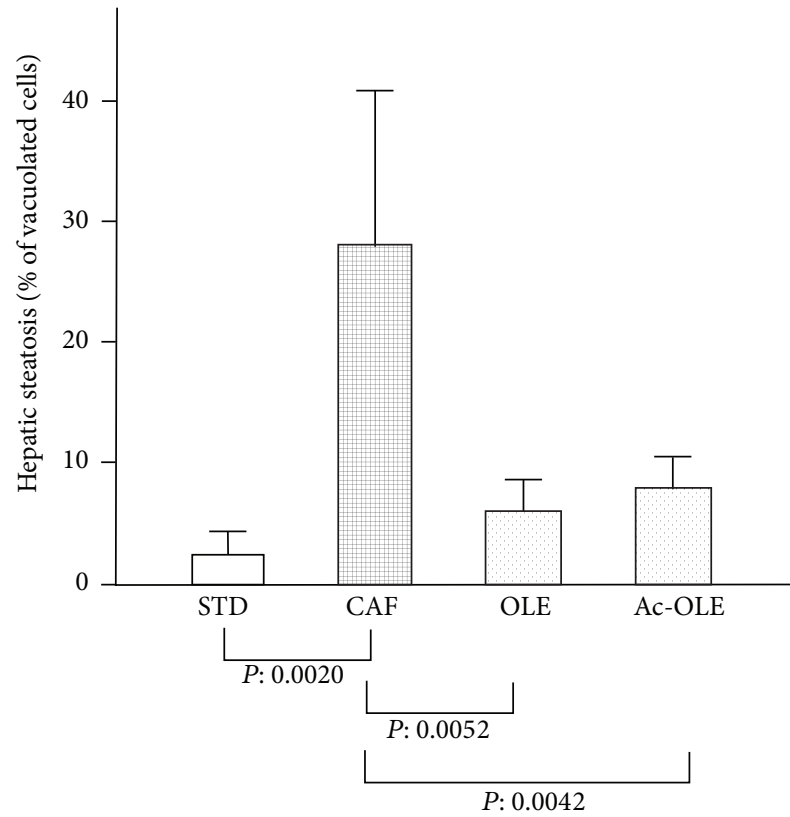

(d)

Figure 2: Effects of Ole and Ac-Ole on liver weight and liver histology. (a) Liver weight was significantly reduced in mice fed with a caf diet supplemented with Ole or Ac-Ole. (b) Relative liver weight. (c) Hematoxylin and eosin staining of liver sections (10x magnification). The liver of mice CAF was rich in fat vacuoles (indicated by the arrow). (d) Quantification of steatosis was expressed as mean of percentage of vacuolated cells calculated for each mouse liver. Values are mean \pm SD. ${ }^{*} P<0.05$ and ${ }^{* *} P<0.01$ compared to STD; ${ }^{\circ} P<0.05$ and ${ }^{\circ} P<0.01$ compared to CAF. 

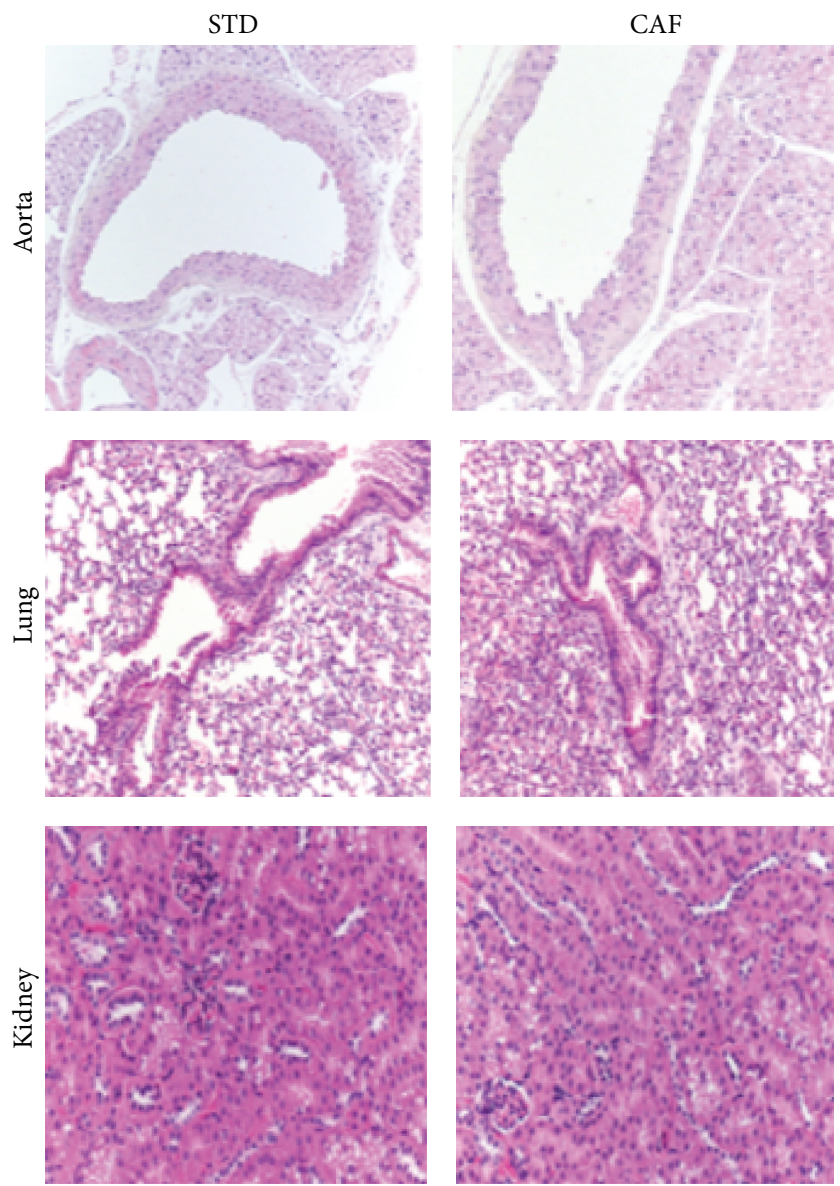

FIgURE 3: Histologic analysis of aorta, lung, and kidney. Hematoxylin and eosin staining of aorta, lung, and kidney sections (10x magnification) from mice fed with std or caf diet.
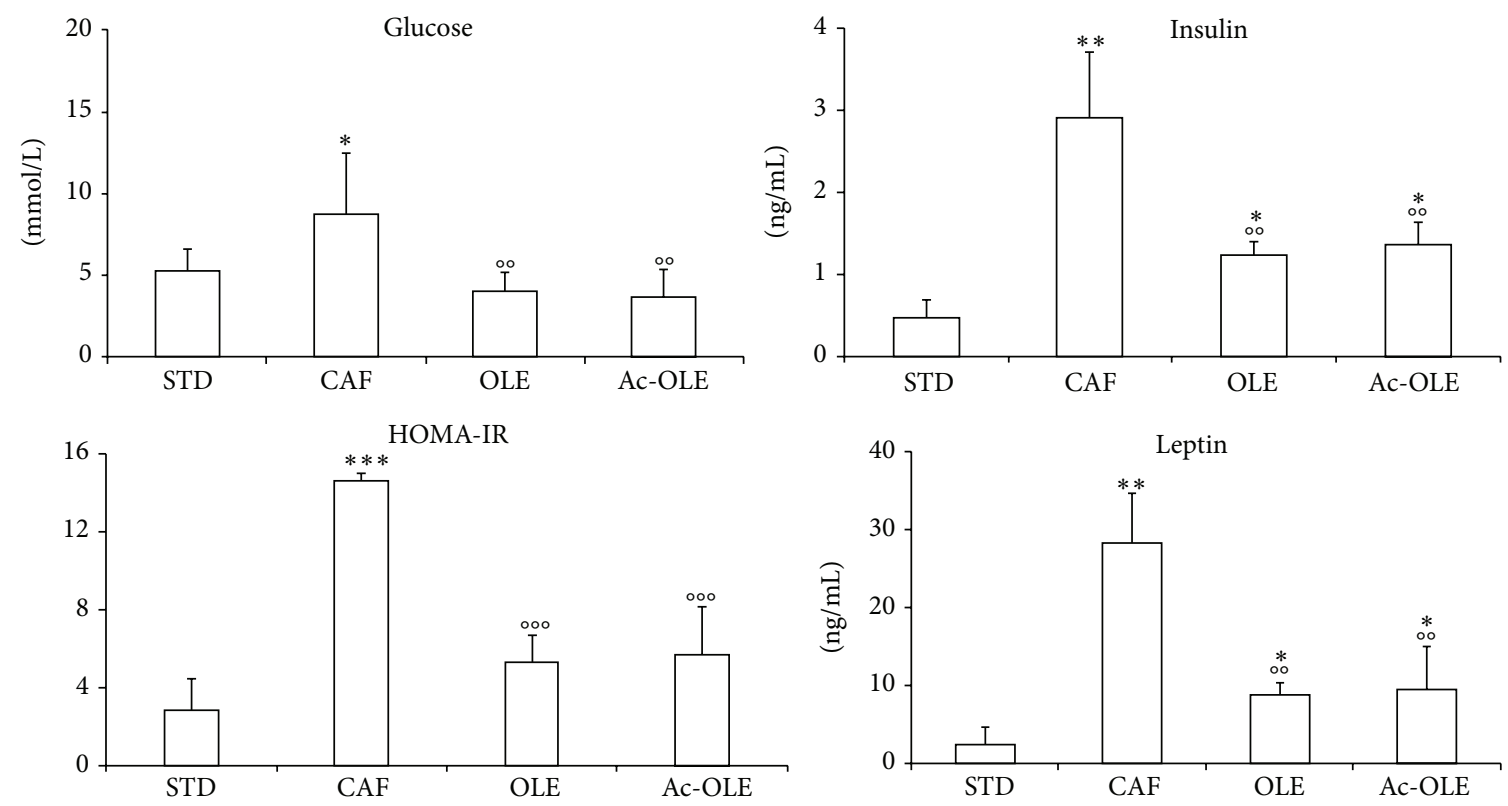

Figure 4: Effects on plasma levels of glucose, insulin, and leptin and evaluation of insulin resistance after treatment with Ole and Ac-Ole. Blood samples were collected as indicated in Methods. Glucose and hormones levels were significantly lower after treatment with Ole and Ac-Ole in animals fed with caf diet. Homeostasis model assessment of insulin resistance (HOMA-IR) was calculated as indicated in Methods. Values are mean $\pm \mathrm{SD} .{ }^{*} P<0.05,{ }^{* *} P<0.01$, and ${ }^{* * *} P<0.001$ compared to STD; ${ }^{\circ 0} P<0.01$ and ${ }^{\circ 00} P<0.001$ compared to CAF. 


\section{Discussion}

Polyphenolic compounds of extra virgin olive oil are known to exert a series of beneficial effects, mainly due to their antioxidant activity. A large number of studies that performed in vitro and in vivo experimental models have focused on the activity of Ole revealing a large array of biological effects either when used as single compound or in a mixture of phenolic extracts from olive oil drupes or leaves $[4,6,7,17]$. In view of a possible use in pharmaceutical field as single drug or as additive in the food, we have synthesized and tested in our laboratory a peracetylated derivative of Ole $[23,25$, 26]. Peracetylation and deacetylation occur naturally in cells thanks to the activity of mitochondrial acetyl-CoA dependent enzymes [30-32]. It has been demonstrated that unprotected drugs, such as $\mathrm{N}$-substituted aromatic compounds, are Nacetylated inside the cells in order to limit their adverse biological effects [30]. Similarly, peracetylated natural compounds such as peracetylated resveratrol or peracetylated epigallocatechin-3-gallate can act as prodrugs because they are deacetylated inside the cells [33]. Since peracetylation improves the chemical stability and permeability without affecting the transportation pathway through the membrane, the intracellular deacetylation results in an augmented dose of unprotected natural active principle in the intracellular medium $[25,26,32]$.

We have previously reported the beneficial effects of Ole and Ac-Ole in vitro. In this study, they have been investigated in vivo to analyze the effects versus the metabolic alterations caused by a caf diet. This diet is composed of a mixture of fat and carbohydrates and resembles in a better way the Western type food habits compared to traditional lard-based high fat diet [27]. Indeed, mice fed with a caf diet develop metabolic syndrome more severely than those fed with high fat diet [34].

In our study, mice fed with this diet for 15 weeks presented an increased body weight associated with histological signs of hepatic steatosis, high serum levels of total and LDLcholesterol and hyperinsulinemia. Only slight changes and inside the normal range levels were observed for transaminases, probably due to the limited period of exposure to this diet, as already reported in the other study [22]. Interestingly, the presence in CAF mice of an increase in insulin levels seems to reproduce the well-known phase of hyperinsulinemic normoglycemia observed in obese individuals before the development of diabetes mellitus 2 (DM2). In our caf fed mice, administration of $0.037 \mathrm{mmol} / \mathrm{kg} /$ day of Ole and $0.025 \mathrm{mmol} / \mathrm{kg} / \mathrm{day}$ of Ac-Ole, determined the following: (a) reduction in the body weight increase due to minor accumulation of fat in the abdominal adipose tissue, with unchanged food intake gain; (b) significant decrease of macroscopic and microscopic steatosis; (c) lower levels of serum lipids, with normalization of the levels of LDLcholesterol; (d) correction of elevated glucose plasmatic levels without increase in insulin levels, associated with normal HOMA-IR.

In a recent study, van der Stelt et al. [35] demonstrated that high dose of Ole supplementation $(758 \mathrm{mg} / \mathrm{kg}$ body weight) in mice fed with a high fat diet resulted in decrease of body weight, serum lipids, hepatic lipid accumulation, and liver weight after 12 weeks of treatment. In our study, the same results were observed using a much lower dose of Ole or Ac-Ole $(20 \mathrm{mg} / \mathrm{kg}$ body weight). Prevention of body weight increase in animals (mice or rats) fed with high fat diet without acting on food intake was also reported using a similar dosage of Ole in similar experimental conditions [36, 37]. As in our study, no action on food intake was observed when Ole was added as single compound, while a decrease was detected when olive leaf phenolic extracts were tested [38], probably owing to the presence of apigenin $[39,40]$. Also, the caloric intake of OLE and Ac-OLE groups was significantly higher with respect to STD but they did not gain weight; we may hypothesize that Ole and Ac-Ole act by increasing the energy expenditure and/or reducing energy retention, by regulating the expression of genes involved in adipogenesis and thermogenesis [38]. Our present data showed that the same prevention of body weight increase was obtained with lower doses of Ac-Ole ( 0.025 versus $0.037 \mathrm{mmol} / \mathrm{kg}$ of Ole). Further studies on the gene expression profile modified by both compounds will help to identify the molecular pathways involved in such an effect. Also prevention of hepatic steatosis exerted by Ole has already been described in animal models $[21,22]$. As for the protective action on body weight increase, an identical effect was obtained in our experimental model using Ac-Ole. Again, as for the anti-flogistic and in vitro antineoplastic effects $[25,26,41,42]$, this semisynthetic compound demonstrated high efficacy.

The most interesting findings of the present study, in our opinion, appear to be those regarding the metabolic effects of the compounds. In our experimental model, exposure to a caf diet determined a significant increase in both total and LDL-cholesterol levels. Ole and Ac-Ole treatment was able to partially prevent such effects and, most importantly, normalize the LDL-cholesterol levels. Andreadou et al. [43, 44] demonstrated that Ole $(20 \mathrm{mg} / \mathrm{kg})$ decreased cholesterol and triglycerides levels in hypercholesterolemic rabbits. Instead, we found no significant changes in triglycerides, probably because in this strain of mice treatments longer than 15 weeks are required to get such alteration [22].

Absence of significant modifications was noted in caf fed mice regarding the glycemic profile. However, insulin levels resulting are higher than mice fed with std diet, so that we may suppose that it was the parallel increase of insulin secretion which counteracted the effects of caf diet. Treatment with Ole and Ac-Ole obtained the same result with levels of insulin maintained in the normal range, with normalization of the values of HOMA-IR. In the absence of euglycemic hyperinsulinemic clamp, it is not possible to fully demonstrate the occurrence of an increased insulin sensitivity, as postulated in other studies using olive leaf polyphenols [19]. Another possibility is that normal insulin levels depend on the lack of body weight increase. In any case, however, we believe that prevention of damage associated with prolonged exposure to hyperinsulinemia that characterizes the pathologies associated with metabolic syndrome, $\mathrm{DM} 2$, and obesity deserves to be underlined. An interference between Ole (or Ac-Ole) and substances present in elements of caf diet (as well as any kind of commercial diet used in this kind of studies) cannot be excluded. 


\section{Conclusions}

In conclusion, these findings demonstrate, in this experimental model of metabolic damage caused by caf diet, the beneficial effects of Ac-Ole, exerted at even lower dose in moles than Ole. Further studies, including the administration of different dosages of Ac-Ole and even for shorter periods will allow characterizing the molecular mechanism involved in such an action, in view of a potential use of this compound for prevention of human metabolic disorders.

\section{Ethical Approval}

The study received the approval by local Institutional Animal Care and Use Committee and was conducted in accordance with current legislation on animal experiments in Italy and with the European Union regulations (O.J. of E.C. L358/1 12/18/1986).

\section{Conflict of Interests}

The authors declare that they have no conflict of interests.

\section{Acknowledgments}

This work was supported by Fondazione Umberto Di Mario ONLUS. Saverio Massimo Lepore is supported by a cofinanced grant from the European Commission, the European Social Fund, and Calabria Region. The authors thank Dr. Nicola Costa for technical assistance in the management of animals.

\section{References}

[1] W. C. Willett, F. Sacks, A. Trichopoulou et al., "Mediterranean diet pyramid: a cultural model for healthy eating," The American Journal of Clinical Nutrition, vol. 61, no. 6, supplement, pp. 1402S-1406S, 1995.

[2] C. L. Huang and B. E. Sumpio, "Olive Oil, the Mediterranean Diet, and Cardiovascular Health," Journal of the American College of Surgeons, vol. 207, no. 3, pp. 407-416, 2008.

[3] R. J. Widmer, A. J. Flammer, L. O. Lerman, and A. Lerman, "The mediterranean diet, its components, and cardiovascular disease," The American Journal of Medicine, vol. 128, no. 3, pp. 229-238, 2015.

[4] S. N. El and S. Karakaya, "Olive tree (Olea europaea) leaves: potential beneficial effects on human health," Nutrition Reviews, vol. 67, no. 11, pp. 632-638, 2009.

[5] S. H. Omar, "Oleuropein in olive and its pharmacological effects," Scientia Pharmaceutica, vol. 78, no. 2, pp. 133-154, 2010.

[6] S. Cicerale, L. Lucas, and R. Keast, "Biological activities of phenolic compounds present in virgin olive oil," International Journal of Molecular Sciences, vol. 11, no. 2, pp. 458-479, 2010.

[7] S. Granados-Principal, J. L. Quiles, C. L. Ramirez-Tortosa, P. Sanchez-Rovira, and M. C. Ramirez-Tortosa, "Hydroxytyrosol: from laboratory investigations to future clinical trials," Nutrition Reviews, vol. 68, no. 4, pp. 191-206, 2010.

[8] S. Bulotta, M. Oliverio, D. Russo, and A. Procopio, "Biological activity of oleuropein and its derivatives," in Natural Products,
K. G. Ramawat and J. M. Mérillon, Eds., pp. 3605-3638, Springer, Heidelberg, Germany, 2013.

[9] B. Barbaro, G. Toietta, R. Maggio et al., "Effects of the olivederived polyphenol oleuropein on human health," International Journal of Molecular Sciences, vol. 15, no. 10, pp. 18508-18524, 2014.

[10] H.-W. Tan, K. L. Tuck, I. Stupans, and P. J. Hayball, "Simultaneous determination of oleuropein and hydroxytyrosol in rat plasma using liquid chromatography with fluorescence detection," Journal of Chromatography B: Analytical Technologies in the Biomedical and Life Sciences, vol. 785, no. 1, pp. 187-191, 2003.

[11] P. Del Boccio, A. Di Deo, A. De Curtis, N. Celli, L. Iacoviello, and D. Rotilio, "Liquid chromatography-tandem mass spectrometry analysis of oleuropein and its metabolite hydroxytyrosol in rat plasma and urine after oral administration," Journal of Chromatography B: Analytical Technologies in the Biomedical and Life Sciences, vol. 785, no. 1, pp. 47-56, 2003.

[12] F. Visioli, A. Poli, and C. Gall, "Antioxidant and other biological activities of phenols from olives and olive oil," Medicinal Research Reviews, vol. 22, no. 1, pp. 65-75, 2002.

[13] S. Cicerale, L. J. Lucas, and R. S. J. Keast, "Antimicrobial, antioxidant and anti-inflammatory phenolic activities in extra virgin olive oil," Current Opinion in Biotechnology, vol. 23, no. 2, pp. 129-135, 2012.

[14] H. Jemai, A. El Feki, and S. Sayadi, "Antidiabetic and antioxidant effects of hydroxytyrosol and oleuropein from olive leaves in alloxan-diabetic rats," Journal of Agricultural and Food Chemistry, vol. 57, no. 19, pp. 8798-8804, 2009.

[15] S. H. Omar, "Cardioprotective and neuroprotective roles of oleuropein in olive," Saudi Pharmaceutical Journal, vol. 18, no. 3, pp. 111-121, 2010.

[16] I. Andreadou, E. Mikros, K. Ioannidis et al., "Oleuropein prevents doxorubicin-induced cardiomyopathy interfering with signaling molecules and cardiomyocyte metabolism," Journal of Molecular and Cellular Cardiology, vol. 69, pp. 4-16, 2014.

[17] S. Bulotta, M. Celano, S. M. Lepore, T. Montalcini, A. Pujia, and D. Russo, "Beneficial effects of the olive oil phenolic components oleuropein and hydroxytyrosol: focus on protection against cardiovascular and metabolic diseases," Journal of Translational Medicine, vol. 12, no. 1, article 219, 2014.

[18] M. Gonzalez, A. Zarzuelo, M. J. Gamez, M. P. Utrilla, J. Jimenez, and I. Osuna, "Hypoglycemic activity of olive leaf," Planta Medica, vol. 58, no. 6, pp. 513-515, 1992.

[19] M. de Bock, J. G. B. Derraik, C. M. Brennan et al., "Olive (Olea europaea L.) leaf polyphenols improve insulin sensitivity in middle-aged overweight men: a randomized, placebocontrolled, crossover trial," PLoS ONE, vol. 8, no. 3, Article ID e57622, 2013.

[20] Y. Kim, Y. Choi, and T. Park, "Hepatoprotective effect of oleuropein in mice: mechanisms uncovered by gene expression profiling," Biotechnology Journal, vol. 5, no. 9, pp. 950-960, 2010.

[21] S. Park, Y. Choi, S.-J. Um, S. K. Yoon, and T. Park, "Oleuropein attenuates hepatic steatosis induced by high-fat diet in mice," Journal of Hepatology, vol. 54, no. 5, pp. 984-993, 2011.

[22] S. W. Kim, W. Hur, T. Z. Li et al., "Oleuropein prevents the progression of steatohepatitis to hepatic fibrosis induced by a high-fat diet in mice," Experimental and Molecular Medicine, vol. 46, no. 4, article e92, 2014.

[23] A. Procopio, G. Sindona, M. Gaspari, N. Costa, and M. Nardi, "Chemical catalytic method for the peracylation of oleuropein and its products of hydrolysis," International Patent no. PCT/ IT2008/000303, 2008. 
[24] A. Procopio, S. Alcaro, M. Nardi et al., "Synthesis, biological evaluation, and molecular modeling of oleuropein and its semisynthetic derivatives as cyclooxygenase inhibitors," Journal of Agricultural and Food Chemistry, vol. 57, no. 23, pp. 11161$11167,2009$.

[25] S. Bulotta, R. Corradino, M. Celano et al., "Antiproliferative and antioxidant effects on breast cancer cells of oleuropein and its semisynthetic peracetylated derivatives," Food Chemistry, vol. 127, no. 4, pp. 1609-1614, 2011.

[26] S. Bulotta, R. Corradino, M. Celano et al., "Antioxidant and antigrowth action of peracetylated oleuropein in thyroid cancer cells," Journal of Molecular Endocrinology, vol. 51, no. 1, pp. 181189, 2013.

[27] B. P. Sampey, A. M. Vanhoose, H. M. Winfield et al., "Cafeteria diet is a robust model of human metabolic syndrome with liver and adipose inflammation: comparison to high-fat diet," Obesity, vol. 19, no. 6, pp. 1109-1117, 2011.

[28] J. H. Yang, H. S. Lim, and Y. R. Heo, “Sasa borealis leaves extract improves insulin resistance by modulating inflammatory cytokine secretion in high fat diet-induced obese C57/BL6J mice," Nutrition Research and Practice, vol. 4, no. 2, pp. 99-105, 2010.

[29] G. Gomori, "A rapid one-step trichrome stain," American Journal of Clinical Pathology, vol. 20, no. 7, pp. 661-664, 1950.

[30] C. M. King and I. B. Glowinski, "Acetylation, deacetylation and acyltransfer," Environmental Health Perspectives, vol. 49, pp. 43$50,1983$.

[31] J. D. Lambert, S. Sang, J. Hong et al., "Peracetylation as a means of enhancing in vitro bioactivity and bioavailability of epigallocatechin-3-gallate," Drug Metabolism and Disposition, vol. 34, no. 12, pp. 2111-2116, 2006.

[32] D. Colin, A. Lancon, D. Delmas et al., "Antiproliferative activities of resveratrol and related compounds in human hepatocyte derived HepG2 cells are associated with biochemical cell disturbance revealed by fluorescence analyses," Biochimie, vol. 90, no. 11-12, pp. 1674-1684, 2008.

[33] S. C. Lee, W. K. Chan, and T. W. Lee, "Effect of a prodrug of the green tea polyphenol (-)-epigallocatechin-3-gallate on the growth of androgen-independent prostate cancer in vivo," Nutrition and Cancer, vol. 60, no. 4, pp. 483-491, 2008.

[34] T. S. Higa, A. V. Spinola, M. H. Fonseca-Alaniz, and F. S. Anna Evangelista, "Comparison between cafeteria and high-fat diets in the induction of metabolic dysfunction in mice," International Journal of Physiology, Pathophysiology and Pharmacology, vol. 6, no. 1, pp. 47-54, 2014.

[35] I. van der Stelt, E. F. Hoek-van den Hil, H. J. Swarts et al., "Nutraceutical oleuropein supplementation prevents high fat diet-induced adiposity in mice," Journal of Functional Foods, vol. 14, pp. 702-715, 2015.

[36] H. Poudyal, F. Campbell, and L. Brown, "Olive leaf extract attenuates cardiac, hepatic, and metabolic changes in high carbohydrate, high fat-fed rats," Journal of Nutrition, vol. 140, no. 5, pp. 946-953, 2010.

[37] N. Kuem, S. J. Song, R. Yu, J. W. Yun, and T. Park, “Oleuropein attenuates visceral adiposity in high-fat diet-induced obese mice through the modulation of WNT10b- and galaninmediated signalings," Molecular Nutrition \& Food Research, vol. 58, no. 11, pp. 2166-2176, 2014.

[38] Y. Shen, S. J. Song, N. Keum, and T. Park, "Olive leaf extract attenuates obesity in high-fat diet-fed mice by modulating the expression of molecules involved in adipogenesis and thermogenesis," Evidence-Based Complementary and Alternative Medicine, vol. 2014, Article ID 971890, 12 pages, 2014.

[39] O. Benavente-García, J. Castillo, J. Lorente, A. Ortuño, and J. A. Del Rio, "Antioxidant activity of phenolics extracted from Olea europaea L. leaves," Food Chemistry, vol. 68, no. 4, pp. 457-462, 2000.

[40] H.-J. Myoung, G. Kim, and K.-W. Nam, "Apigenin isolated from the seeds of Perilla frutescens britton var crispa (Benth.) inhibits food intake in C57BL/6J mice," Archives of Pharmacal Research, vol. 33, no. 11, pp. 1741-1746, 2010.

[41] D. Impellizzeri, E. Esposito, E. Mazzon et al., "The effects of oleuropein aglycone, an olive oil compound, in a mouse model of carrageenan-induced pleurisy," Clinical Nutrition, vol. 30, no. 4, pp. 533-540, 2011.

[42] D. Impellizzeri, E. Esposito, E. Mazzon et al., "The effects of a polyphenol present in olive oil, oleuropein aglycone, in an experimental model of spinal cord injury in mice," Biochemical Pharmacology, vol. 83, no. 10, pp. 1413-1426, 2012.

[43] I. Andreadou, E. K. Iliodromitis, E. Mikros et al., "The olive constituent oleuropein exhibits anti-ischemic, antioxidative, and hypolipidemic effects in anesthetized rabbits," Journal of Nutrition, vol. 136, no. 8, pp. 2213-2219, 2006.

[44] I. Andreadou, D. Benaki, P. Efentakis et al., "The natural olive constituent oleuropein induces nutritional cardioprotection in normal and cholesterol-fed rabbits: comparison with preconditioning," Planta Medica, vol. 81, no. 8, pp. 655-663, 2015. 


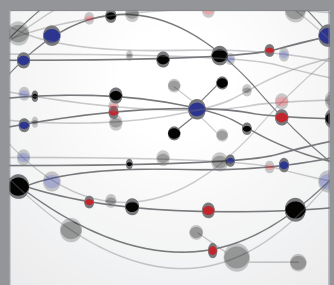

The Scientific World Journal
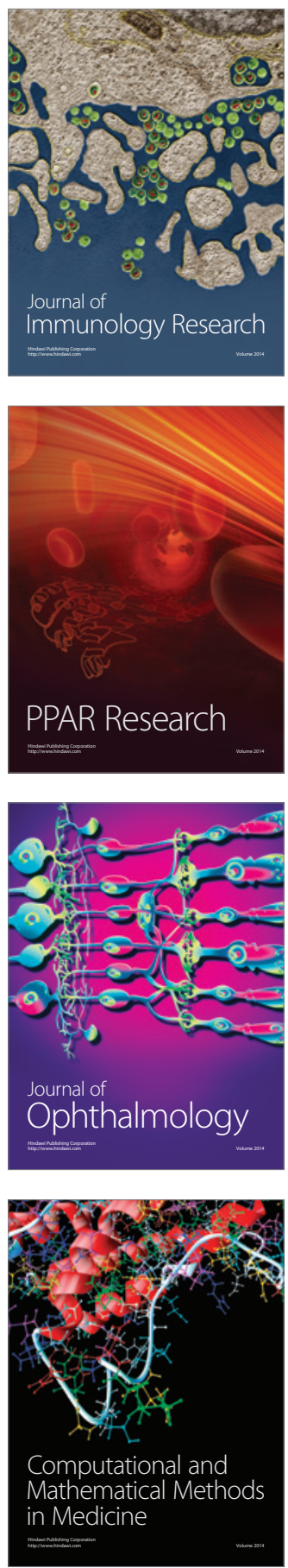

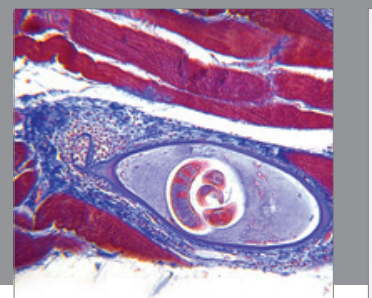

Gastroenterology

Research and Practice
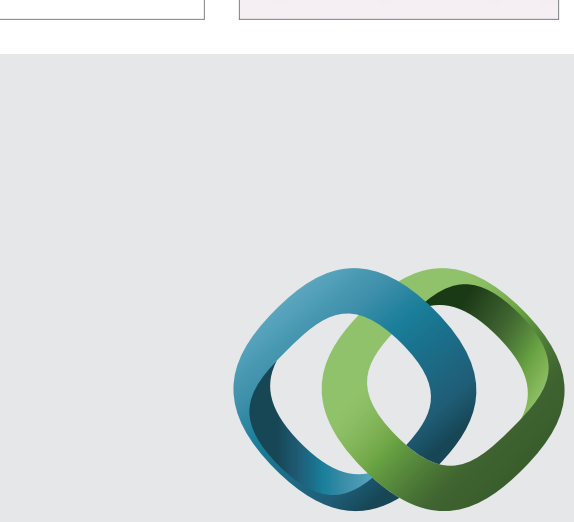

\section{Hindawi}

Submit your manuscripts at

http://www.hindawi.com
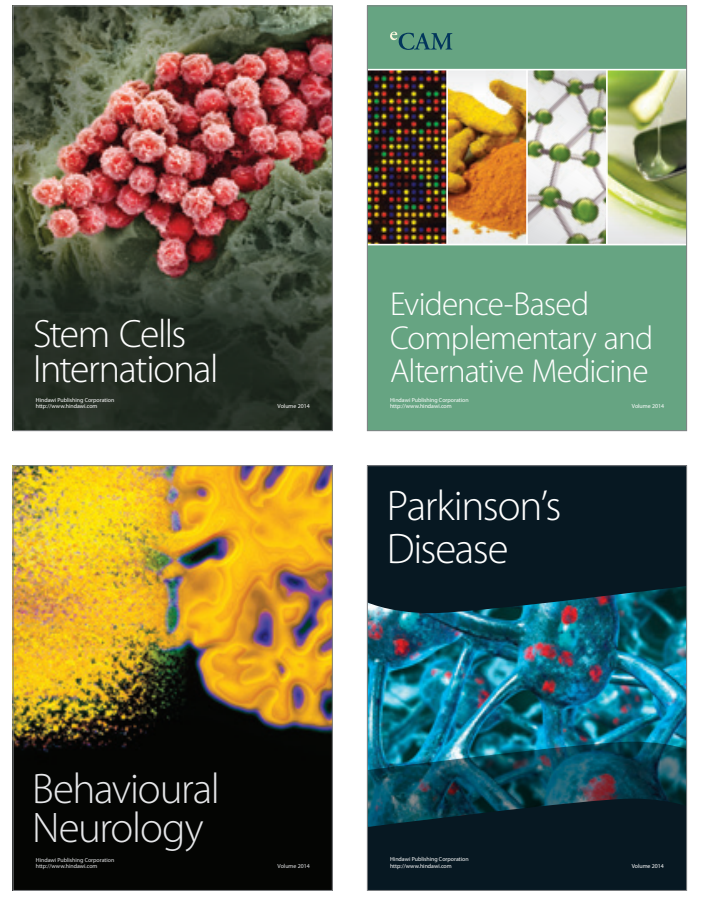
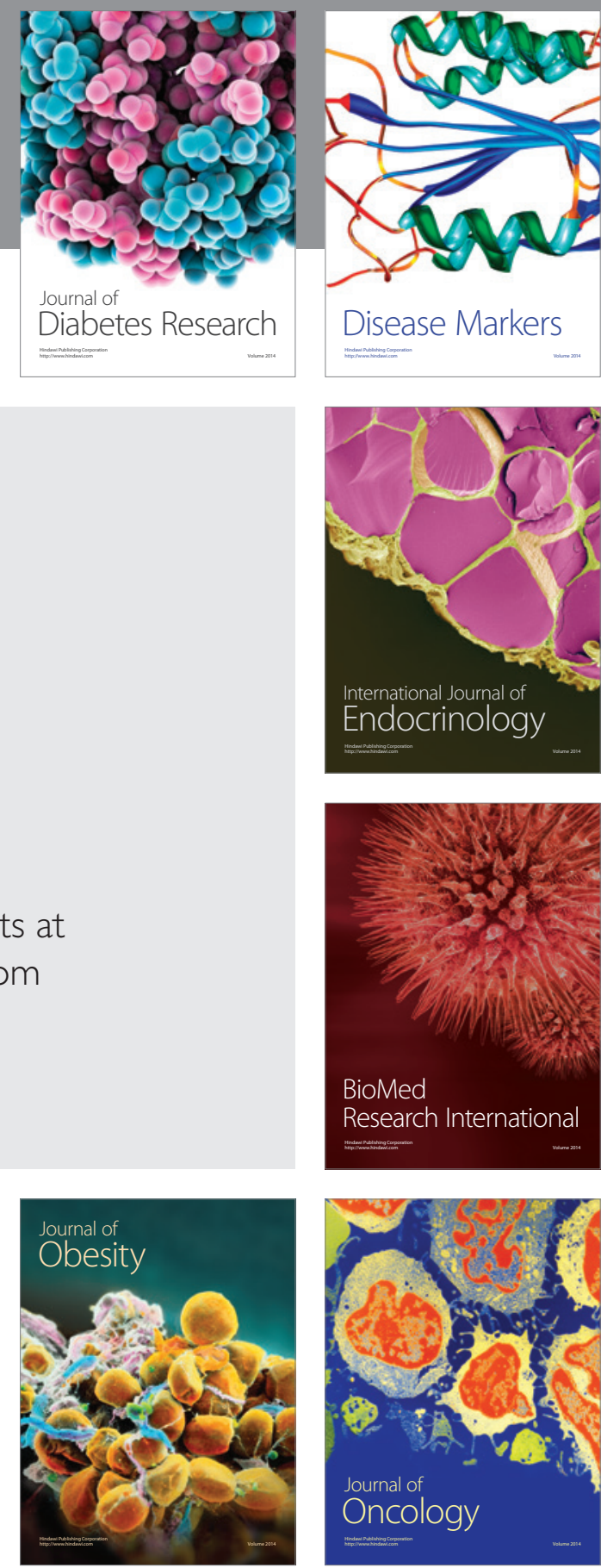

Disease Markers
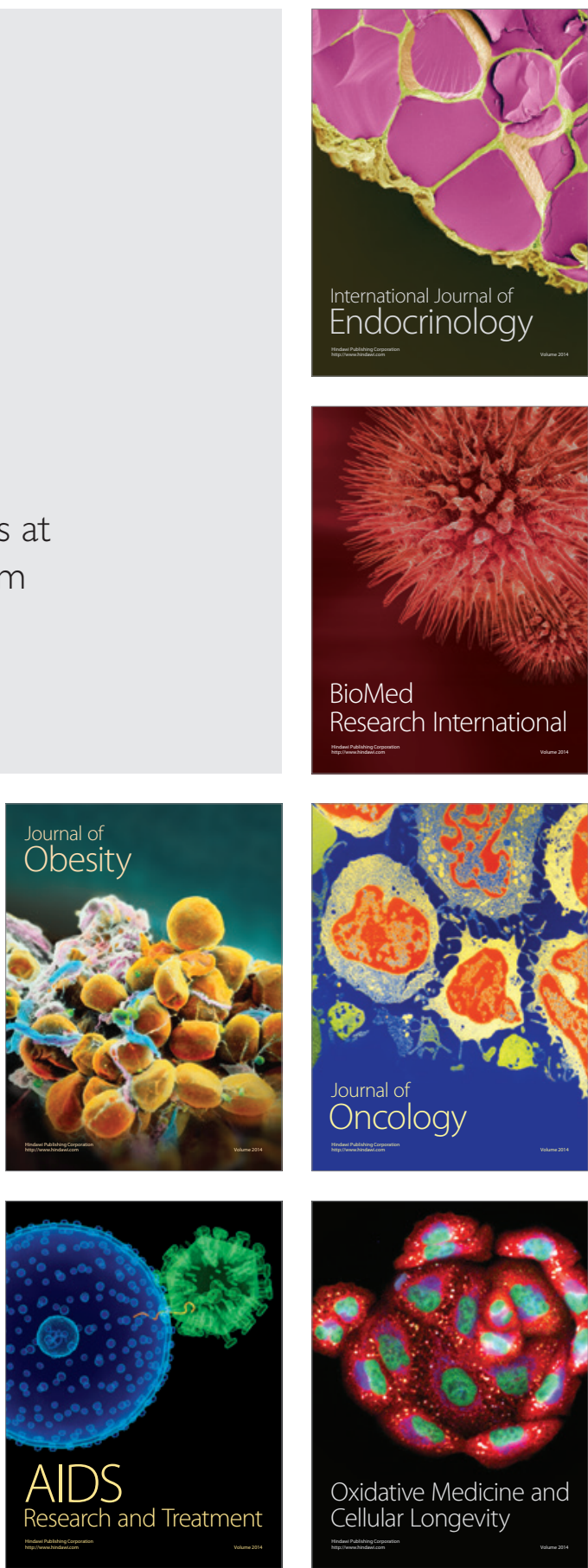